Stéphane ANDRÉ, Le Roman français contemporain à l'épreuve du tourisme : (dé)jouer le stéréotype pour renouer avec le voyage

Thèse de doctorat en littérature et civilisation françaises de l'Université Sorbonne Nouvelle-Paris 3, dirigée par Bruno Blanckeman (soutenue le 4 décembre 2017)

The contemporary French novel confronted to tourism (1990-2010). Playing with stereotypes to go back to travelling

\title{
Stéphane André
}

\section{OpenEdition}

\section{Journals}

Édition électronique

URL : http://journals.openedition.org/tourisme/2209

DOI : $10.4000 /$ tourisme.2209

ISSN : 2492-7503

Éditeur

Éditions touristiques européennes

Référence électronique

Stéphane André, «Stéphane ANDRÉ, Le Roman français contemporain à l'épreuve du tourisme

(dé)jouer le stéréotype pour renouer avec le voyage », Mondes du Tourisme [En ligne], 15 | 2019, mis en ligne le 01 juin 2019, consulté le 25 septembre 2020. URL : http://journals.openedition.org/tourisme/ 2209 ; DOI : https://doi.org/10.4000/tourisme.2209

Ce document a été généré automatiquement le 25 septembre 2020

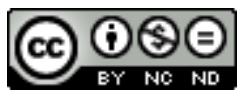

Mondes du tourisme est mis à disposition selon les termes de la licence Creative Commons Attribution - Pas d'Utilisation Commerciale - Pas de Modification 4.0 International. 


\section{Stéphane ANDRÉ, Le Roman français contemporain à l'épreuve du tourisme : (dé)jouer le stéréotype pour renouer avec le voyage}

Thèse de doctorat en littérature et civilisation françaises de l'Université Sorbonne Nouvelle-Paris 3, dirigée par Bruno Blanckeman (soutenue le 4 décembre 2017)

The contemporary French novel confronted to tourism (1990-2010). Playing with stereotypes to go back to travelling

Stéphane André

\section{NOTE DE L'AUTEUR}

Thèse accessible en ligne : https://tel.archives-ouvertes.fr/tel-01772374/document ; publication à venir

1 En raison de son caractère supposément prévisible et stéréotypé, le tourisme a longtemps été relégué aux confins de la recherche littéraire : il n'existerait de voyage authentique qu'en marge des réseaux organisés. Cette conviction est si profondément enracinée que très peu d'ouvrages se proposent d'examiner l'influence qu'exerce aujourd'hui le tourisme sur la littérature. Cette frilosité apparaît étonnante tant elle contraste avec l'abondance des travaux menés dans le champ des sciences humaines et sociales; elle apparait d'autant plus injustifiée qu'elle n'est guère partagée par les écrivains eux-mêmes, qui, de longue date, ont entretenu un rapport très conscient avec le tourisme : fût-ce pour le dénigrer, il y a longtemps qu'ils s'en inspirent. Ce constat rendait nécessaire l'étude de l'influence qu'exerce le tourisme sur le champ des écritures contemporaines. Nous nous sommes efforcé de mener ce questionnement à 
partir d'une trentaine de romans choisis en raison de leur caractère délibérément éclectique, mêlant des auteurs jouissant d'une grande visibilité (à l'instar de Michel Houellebecq, Mathias Énard ou Lydie Salvayre) et des romanciers à l'audience plus confidentielle. Si cette thèse adopte une perspective synchronique en privilégiant la littérature de l'extrême contemporain - et singulièrement la période 1990-2010 -, elle s'efforce aussi, à chaque fois que possible, de mettre en perspective les écritures d'aujourd'hui avec une longue tradition antitouristique, qui permet de réfléchir à la manière dont les littératures d'hier et d'aujourd'hui se nourrissent du tourisme et se renouvellent à son contact.

\section{Roman et tourisme, une parenté insoupçonnée}

2 Cet examen s'avère d'autant plus stimulant qu'il révèle tout d'abord une étrange proximité entre genre romanesque et pratique touristique : l'un et l'autre sont, en effet, deux produits de la modernité européenne; tous deux proposent une expérience cognitive en partie adossée au réel et en partie nourrie des apports de la fiction; ils contribuent également à l'élaboration d'un décor et modalisent le rapport que nous entretenons avec le monde extérieur. L'un et l'autre, enfin, sont étroitement liés à la diffusion de l'écrit : ainsi que le note Catherine Bertho-Lavenir,

[t]oute l'histoire du tourisme est le récit croisé de transformations arrivées dans les techniques du transport et les techniques d'écriture, dans l'économie du chemin de fer et dans celle de l'imprimerie, dans l'expérience de voyage et les formes du récit. (Bertho-Lavenir, 1999, p. 43)

3 Ce cousinage est loin d'être anecdotique ; il se révèle même étonnamment productif dès lors qu'il ouvre à l'écrivain la possibilité d'un questionnement réflexif, qui tend à faire de l'aménagement touristique une métaphore de la création romanesque.

L'attention que portent les auteurs d'aujourd'hui à cette question est d'autant plus grande qu'à bien des égards le tourisme radicalise les travers de nos sociétés contemporaines: en multipliant les dispositifs spécifiques, le secteur touristique participe d'une entreprise de déréalisation qui a beaucoup à voir avec la mise en spectacle décrite en son temps par Guy Debord. Ainsi que le note Bruno Blanckeman, fin connaisseur des littératures de l'extrême contemporain,

[1]es nouvelles donnes de l'image, technologiques - monde du virtuel - et

idéologiques - société-spectacle - intéressent la littérature en cela qu'elles

brouillent les repères distinctifs de la réalité et de sa simulation, confondent le sens

$\mathrm{du}$ réel et son absence, identifient la conscience du vrai et la perception du factice.

(Blanckeman, 2000, p. 205)

5 C'est cette frontière entre réalité et fiction que nous nous proposons d'explorer, en abordant successivement la représentation que le roman donne du touriste, du tourisme et du touristique (en tant que regard porté sur le monde extérieur).

\section{Le touriste, incarnation d'un trouble identitaire très contemporain}

6 Figure longtemps caricaturée, souvent reléguée au statut de personnage secondaire et volontiers moquée pour son inculture et son absence de discernement, le touriste tel qu'il est représenté en littérature depuis le XIX ${ }^{\mathrm{e}}$ siècle - et plus encore dans les années 
1960 - s'est beaucoup complexifié : il y a loin du touriste tel que le décrivait Julien Gracq, Paul Morand ou Marguerite Yourcenar à celui que l'on représente au tournant du XXI ${ }^{e}$ siècle.

7 Le touriste en vient même à constituer une figure privilégiée pour interroger la condition de l'individu postmoderne, personnage clivé, partagé entre des motivations contradictoires. À l'instar du romancier, le voyageur contemporain apparaît bien souvent en porte-à-faux. Ainsi voit-on le protagoniste du roman Oreille rouge d'Éric Chevillard (2005) osciller continûment entre aspiration à la sécurité et quête de l'aventure. Les auteurs contemporains se montrent également beaucoup plus attentifs aux relations complexes que les touristes entretiennent avec leurs semblables, comme en témoignent aussi bien Les belles âmes que Lydie Salvayre fait paraître en 2000 que Plateforme que Michel Houellebecq publie un an plus tard. Les préoccupations que manifestent les voyageurs ne sont d'ailleurs pas sans liens avec celles qu'éprouvent les écrivains eux-mêmes, désireux qu'ils sont de s'inspirer de leurs devanciers tout en se démarquant de leurs contemporains. Le recensement des lectures auxquelles se livrent les personnages de touristes offre d'ailleurs un aperçu très riche des références dont (se) jouent les auteurs d'aujourd'hui. Ainsi envisagé, le touriste se prête idéalement à la mise en abyme de la figure du romancier.

\section{Microcosme touristique et territoire romanesque}

8 Le tourisme ne se réduit cependant pas aux seuls touristes: il suppose également l'existence de tout un secteur d'activités qui, à l'instar du roman, constitue une manière de monde clos que caractérise une temporalité, des espaces, des figures et un langage qui lui sont propres: ne parle-t-on pas souvent de "bulle touristique»? C'est cette hétérotopie (au sens où l'entendait Michel Foucault, 1994) que les écrivains s'efforcent à leur manière d'investir et de questionner: plutôt que de prétendre occulter les dispositifs touristiques qui conditionnent aujourd'hui le voyage (occultation en trompe-l'œil qui participerait elle-même d'une logique touristique), nombre d'auteurs s'efforcent au contraire de leur donner une plus grande visibilité.

Cette réappropriation littéraire du secteur touristique prend des formes très variées. Si certains romanciers se montrent surtout attentifs au temps du voyage et à la manière dont celui-ci interfère avec le rythme de la fiction romanesque - phénomène observable notamment dans Zone de Mathias Énard, paru en 2008 -, d'autres préfèrent porter leur attention sur les lieux du tourisme, à l'image d'Olivier Rolin, qui s'inspire de l'espace clos de la chambre d'hôtel pour donner forme à son roman Suite à l'hôtel Crystal (2006), ou de Jean Echenoz, qui propose une représentation du paradis, du purgatoire ou des enfers directement inspirée d'un village vacances, d'un palace des années 1900 ou d'un hôtel bas de gamme (Au Piano, 2003). Les professionnels du tourisme ne sont pas en reste, comme en témoigne l'œuvre de Jean-Philippe Toussaint, qui tend à les assimiler à des personnages de fiction. Quant au langage souvent prévisible des guides touristiques, ils font les délices d'auteurs comme Éric Chevillard, qui s'emploient à en subvertir le caractère prévisible tout en dénonçant par la même occasion les lieux communs du romanesque (Préhistoire, 1994). 


\section{Une interface pour appréhender le monde réel}

Si le tourisme tel qu'il est représenté dans la fiction romanesque contemporaine apparaît à ce point complexe, c'est qu'il constitue une remarquable grille de lecture à travers laquelle entrevoir notre contemporanéité. Le rôle d'interface qu'il joue entre le voyageur et les territoires qu'il parcourt fait du tourisme un support privilégié pour penser indirectement le travail du romancier : ce dernier ne propose-t-il pas, à travers le prisme de son écriture, une certaine vision du monde, de l'Histoire, des langues et de l'altérité ? Cette proximité n'est pas sans conséquence : elle fait de l'écrivain celui qui peut, mieux que quiconque, interroger la facticité de la mise en scène touristique. Ainsi que le note Christine Jérusalem au sujet de Jean Echenoz (mais la remarque vaut pour beaucoup d'autres auteurs),

[1]es trompe-l'œil bâtis par l'écrivain ne servent pas à produire une illusion mais à

l'exhiber. Le roman ne met pas seulement en scène la « société du spectacle », il en montre les coulisses, les couleurs et la bande-son. En somme, tout ce qui le désigne

comme simulacre. (Jérusalem, 2005, p. 90)

11 À cet égard, le roman contemporain semble renouer avec une tradition caustique et se révèle bien souvent sans concession : la tentation du pittoresque, à laquelle ont parfois succombé les écrivains, se voit subvertie ou tournée en dérision par Jean-Philippe Toussaint (Fuir, 2005); Mathias Énard comme Michel Houellebecq interrogent la propension qu'aurait le tourisme à enseigner l'Histoire ; Olivier Rolin et Jean-Philippe Toussaint soulignent le potentiel comique que recèlent bien des situations typiquement touristiques; quant à Michel Houellebecq, il revisite à sa façon le tourisme sexuel tandis que Jean-Philippe Toussaint ou Éric Chevillard questionnent et contestent plus largement la représentation souvent stéréotypée que le tourisme s'emploie à donner de l'autochtone.

\section{Entre dévoilement et subversion}

Il ressort de cet examen la sensation d'une étonnante vitalité : après des décennies de critique antitouristique, le roman contemporain s'efforce de réintégrer le «horschamp » du tourisme. Il ne s'agit pas (ou plus seulement) pour l'écrivain d'en contester le bien-fondé, mais d'en mesurer l'emprise et de se repenser lui-même à son contact. Cette dynamique, qui transcende les lignes éditoriales (bien que les Éditions de Minuit y jouent un rôle souvent prépondérant) et la question du degré de littérarité (elle concerne aussi bien des auteurs grand public que des écrivains plus confidentiels), invite à repenser en profondeur l'étude des littératures du voyage. La parution récente d'un roman comme Autour du monde de Laurent Mauvignier (2014) conduit même à se demander si l'on n'assiste pas à l'émergence d'une catégorie nouvelle que constituerait le roman de tourisme, laquelle, par des moyens qui lui sont propres, s'emploierait à investir et parfois à subvertir les logiques touristiques. Autant d'éléments qui devraient inviter chercheurs en littérature comme en sciences sociales à mieux prendre en compte les représentations que le roman contemporain donne du tourisme. 


\section{BIBLIOGRAPHIE}

Catherine BERTHO-LAVENIR, La roue et le stylo. Comment nous sommes devenus touristes, Odile Jacob, coll. « Le Champ médiologique », 1999.

Bruno BLANCKEMAN, Les récits indécidables : Jean Echenoz, Hervé Guibert, Pascal Quignard, Presses universitaires du Septentrion, coll. « Perspectives », 2000.

Michel Foucault, Dits et écrits IV (1954-1988), Gallimard, coll. « Bibliothèque des sciences humaines », 1994.

Christine JÉRUSALEM, Jean Echenoz : géographies du vide, Publications de l'Université de Saint-

Étienne, Centre interdisciplinaire d'étude et de recherche sur l'expression contemporaine, 2005.

INDEX

Mots-clés : tourisme, littérature, littérature contemporaine, roman, guides touristiques 